\title{
ON THE SPECTRA OF GROUP COMMUTATORS
}

\section{R. PUTNAM AND A. WINTNER}

1. All operators will be supposed bounded, linear (everywhere defined) over a Hilbert space. By the commutator, $C$, of two operators $A$ and $B$ will be meant the operator

$$
C=A B-B A \text {. }
$$

A conjecture of Kaplansky, cited in [4], that if $A$ commutes with $C$, so that

$$
A C=C A,
$$

then

$$
\operatorname{sp}(C)=0 \text { only }
$$

( $\mathrm{sp}=$ spectrum), was recently proved by Kleinecke [6] and Shirokov [9]. Earlier proofs of (3) under the additional restriction that $B$ also commutes with $C$ were given in [8] and [10]. In case $A$ and $B$ are nonsingular, so that 0 does not belong to the spectrum of either $A$ or $B$, one can consider not only the "ring" commutator (1) but also the "group" commutator $D$ defined by

$$
D=A B A^{-1} B^{-1} \text {. }
$$

The object of the present note is to give a condition under which (1), (2) and (4) imply

$$
\operatorname{sp}(D)=1 \text { only. }
$$

It is clear that (5) is to (4) as (3) is to (1). There will be proved the following

Theorem 1. Let $A$ and $B$ be nonsingular and suppose that (1) satisfies (2). In addition, suppose that $A$ has a logarithm $E$ which commutes with every operator which commutes with $A$, thus

$$
A=e^{E}, \quad A X=X A \Rightarrow E X=X E(X \text { arbitrary }) .
$$

Then (4) satisfies (5).

2. Proof of theorem 1. Relations (1) and (2) imply

$$
e^{t A} B e^{-t A}=B+t C
$$

for all real $t$; cf., e.g., $[2 ; 5] .{ }^{1}$ Since $B+t C=\left(I+t C B^{-1}\right) B$, and since

Received by the editors August 17, 1957.

${ }^{1}$ It is interesting to note that Shirokov's proof begins with this basic relation. 
the left side of the equation (7) is nonsingular for all $t$, it follows that $I+t C B^{-1}$ is nonsingular for all $t$. Hence

$$
\operatorname{sp}\left(C B^{-1}\right)=0 \text { only. }
$$

Next, define $F$ by

$$
F=E B-B E,
$$

where $E$ is a logarithm of $A$ assumed in Theorem 1. A simple calculation shows that, in view of the obvious relation $E A=A E$,

$$
A F-F A=E C-C E .
$$

By (2), and (6), $E C=C E$ and so $A F=F A$. Another application of (6) now leads to

$$
E F=F E .
$$

Hence, exactly as (1) and (2) imply (8), relations (9) and (11) imply

$$
\operatorname{sp}\left(F B^{-1}\right)=0 \text { only. }
$$

Furthermore, corresponding to (7) with $t=1$, one has $e^{E} B e^{-E}$ $=B+F$; hence, by (6), $A B-B A=F A$, that is, $C=F A$. But (12) now implies $\operatorname{sp}\left(C A^{-1} B^{-1}\right)=0$ only. Hence (5) follows and the proof of Theorem 1 is complete.

3. The spectrum of an arbitrary operator $G$ is a bounded, closed set; [11]. Hence, the canonical decomposition of its (open) complement contains a unique unbounded (connected) component. It was shown in [12] that if 0 is in this unbounded component, then $G$ has a logarithm $H$. Moreover, it is clear from the proof that $H$ can be expressed as the sum of a finite number of power series in $G$. In particular, relation (6) holds if $A$ and $E$ are replaced by $G$ and $H$ respectively. Thus, one obtains the following

THEOREM 2. Let $A$ and $B$ be nonsingular and suppose that (1) satisfies (2). In addition suppose that 0 is in the unbounded component of the complement of $s p(A)$. Then (4) satisfies (5).

An obvious consequence of Theorem 2 is the

Corollary. Let $A$ be completely continuous, $B$ be arbitrary, and both $A$ and $B$ be nonsingular. Then, if (1) satisfies (2), (4) satisfies (5).

4. Remarks. It is known that nonsingularity of an operator $G$ is not sufficient to guarantee the existence of a square root and, $a$ fortiori, not sufficient to assure the existence of a logarithm; [3]. However, it will remain undecided whether (2) and the nonsingular- 
ity of $A$ and $B$ are sufficient to imply (5). Those conditions suffice if, for instance, $A$ is completely continuous (by Theorem 2) or if, say, $A$ is normal (this is clear from the observation that the spectral resolution furnishes a logarithm with the desired property of (6)).

It can be remarked also that the implication statement of (6) is not assured by the mere existence of a logarithm, even in the case of finite matrices. This is seen if one notes that the matrix

$$
E=\left(\begin{array}{cc}
0 & 0 \\
0 & 2 \pi i
\end{array}\right)
$$

is a logarithm of the $2 \times 2$ unit matrix, $I$; and that, while $I$ commutes with every $X$, the (nonscalar) matrix $E$ cannot. Of course, $I$ also possesses the logarithm 0 which does satisfy (6).

Finally it can be noted that the implication assertion of relation (6) is used essentially to obtain (11) and that, possibly, the latter may hold even when (6) does not.

\section{REFERENCES}

1. G. Birkhoff, Analytical groups, Trans. Amer. Math. Soc. vol. 43 (1938) pp. 61101.

2. J. E. Campbell, On a law of combination of operators bearing on the theory of continuous transformation groups, Proc. London Math. Soc. vol. 28 (1897) pp. 381390.

3. P. R. Halmos, Günter Lumer and J. J. Schäffer, Square roots of operators, Proc. Amer. Math. Soc. vol. 4 (1953) pp. 142-149. 198.

4. - Commutators of operators, II, Amer. J. Math. vol. 76 (1954) pp. 191-

5. F. Hausdorff, Die symbolische Exponentialformel in der Gruppentheorie, Leipzig Berichte vol. 58 (1906) pp. 19-48.

6. D. C. Kleinecke, On operator commutators, Proc. Amer. Math. Soc. vol. 8 (1957) pp. 535-536.

7. G. Lumer, The range of the exponential function, Publ. del Instituto de Mat., Montevideo vol. 3, No. 2, pp. 53-55.

8. C. R. Putnam, On the spectra of commutators, Proc. Amer. Math. Soc. vol. 5 (1954) pp. 929-931.

9. F. V. Shirokov, Proof of a conjecture of Kaplansky, Uspehi Mathematičeskih Nauk vol. 11 (1956) p. 167. 330.

10. I. Vidov, Uber eine Vermutung von Kaplansky, Math. Zeit. vol. 62 (1955) p.

11. A. Wintner, Zur Theorie der beschränkten Bilinearformen, Math. Zeit. vol. 30 (1929) pp. 228-282.

12. - On the logarithms of bounded matrices, Amer. J. Math. vol. 74 (1952) pp. $360-364$.

Purdue University AND

The Johns Hopkins University 